\title{
Regulatory efforts in response to a decade of scientific advancement in bioanalytical methods
}

\author{
Sam H Haidar*,1 \\ ${ }^{1}$ US Food \& Drug Administration, Center for Drug Evaluation and Research, 10903 New Hampshire Ave., Silver Spring, MD 20993, \\ USA \\ *Author for correspondence: Sam.Haidar@fda.hhs.gov
}

\begin{abstract}
"As regulators gain greater experience and understanding of various scientific issues, they are better positioned to exercise flexibility in the interpretation and application of regulations and guidances."
\end{abstract}

First draft submitted: 9 February 2019; Accepted for publication: 21 February 2019; Published online: 4 April 2019

Keywords: Crystal City workshops • method validation • regulated bioanalysis

Advances in science and technology have occurred at a rapid pace over the past decade. Artificial intelligence and self-driving cars, a topic of science fiction fantasy only a few decades ago, have an increasingly significant role in our lives today. It is no wonder then, that over the past decade, big strides have also taken place in the science of bioanalytical methods for small and large molecules. This rapid pace of change has created a challenge to the regulatory environment to adapt accordingly. Prior to discussing in greater detail, a brief historical perspective is in order. The focus will be on analytical methods for small molecules. Biologics and biosimilars are beyond the scope of this commentary.

\section{Historical background}

In January 1999, the US FDA released for public comment the first draft guidance for industry on bioanalytical method validation (BMV). This guidance was finalized and published in May 2001 [1]. Almost 10 years prior, in 1990, the first of a series of workshops held jointly between the FDA and American Association of Pharmaceutical Scientists (AAPS) took place. These meetings came to be known as the Crystal City workshops. The workshop in 1990 was the first major attempt at harmonizing and defining criteria for analytical method validation. Prior to this event, procedures and criteria were not well defined by the scientific community or by regulations. Submissions to regulatory agencies reflected a great deal of variability and subjective application (and interpretation) of procedures for method validation and study sample analysis.

Publication of the BMV guidance in 2001 addressed many of the above concerns [1]. This was accomplished by incorporating the deliberations and consensus reached at two workshops: Analytical Methods Validation: Bioavailability, Bioequivalence and Pharmacokinetic Studies (held in December 1990) [2]; and Bioanalytical Methods Validation: A Revisit With a Decade of Progress (held in January 2000) [3]. It set the procedures for method development and validation to include accuracy, precision, stability, sensitivity, selectivity and reproducibility. For the first time, criteria were established for some of these parameters. For example, during accuracy and precision testing, acceptable values were set at 15\% for concentrations above the lower limit of quantitation (LLOQ), and $20 \%$ for the LLOQ. These standards have been accepted and applied worldwide (small molecules) until our present day.

Since the release of the BMV guidance in 2001, there have been great advances in analytical methods resulting in greater sensitivity, specificity, hybrid platforms incorporating LC-MS/MS and ligand-binding assays, electronic laboratory notebooks and wider use of robotics [4]. In addition to the innovations in science and technology, both industry and regulatory agencies have gained knowledge and experience with analytical methods culminating in 
a better understanding of variables that may impact the results of an analytical procedure, versus others that may not be significant. Assay validation and testing methods have changed accordingly. A prime example is incurred sample reanalysis (or reproducibility [ISR]). This procedure, which was not covered in the 2001 BMV guidance, is currently recommended in the US FDA BMV guidance, published in May 2018, and EMA's BMV guidance, which went into effect on 1 February 2012 [5,6]. ISR and other topics that have come to the forefront of issues discussed by the bioanalytical community and regulatory agencies are examined briefly below.

\section{Incurred sample reanalysis}

The premise behind this recommendation is that spiked matrices constituting quality control (QC) samples and calibration curve (standards) may not always mimic study samples [7,8]. Differences impacting accuracy and precision may be attributed to altered protein binding, reversible metabolism, concomitant medications and other factors. The addition of ISR as a component of method validation has added another level of confidence in the reliability of the analytical method.

Although there was some history with ISR prior to 2006 [7], it became a major topic of discussion at the 3rd AAPS/FDA Workshop (Crystal City III), which took place in May 2006 [8]. It was further elaborated on and sampling strategy defined in the following workshop: The AAPS Workshop 2008 on Current Topics in GLP Bioanalysis: Assay Reproducibility for Incurred Samples (Crystal City IV) [9]. If we compare the FDA's BMV guidance published in May 2018 to the BMV guidance finalized in 2001, the recommendation for ISR testing may be the single most significant addition to bioanalytical method validation.

\section{Different anti-coagulants}

The 2001 BMV (US FDA) recommended partial validation for a method developed using plasma with one anticoagulant before it could be applied to samples with a different coagulant. The justification for the additional validation lies in the fact that different anticoagulants can alter the chemistry (e.g., $\mathrm{pH}$, protein binding) of the blood samples thus impacting sample processing and analysis. Later, it was recognized by industry and regulators that this issue has two components: first, dealing with totally different anticoagulants such as heparin and $\mathrm{K}_{2} \mathrm{EDTA}$, and second, different counter ions of the same anticoagulant, for example, $\mathrm{K}_{2}$ EDTA versus $\mathrm{K}_{3} \mathrm{EDTA}$. In the first case, there is wide agreement that method validation conducted with one anticoagulant could not be used for samples containing a different anticoagulant without additional validation. This concern was debated at the Crystal City III Workshop in 2006. In the conference report published in 2007, it was reported that there was agreement about the need to partially validate first case above, although there was no consensus on the degree of cross-validation needed when samples had the same anticoagulant but different counter ions (second case) [8]. As an example of counter ions in anticoagulants, they cited a method validated on samples containing sodium heparin being applied to samples with lithium heparin. Additional discussions followed in subsequent workshops. By 2018, US FDA took the position, as stated in the BMV guidance (May 2018) that partial validation is recommended in cases of different anticoagulants. For validation and testing involving counter ions of the same anticoagulant, the consensus has been that insignificant impact is expected. Barring data to the contrary, additional validation work would not be needed.

\section{Stability of co-administered meds or fixed dose combination}

This topic also has received its share of discussion and controversy, although in more recent years. The 2001 BMV guidance covers co-administered medications in selectivity testing, but nothing in terms of long-term stability. This suggests that this concern was not identified or deemed significant in the 10 years following the first Crystal City workshop in 1990. The Crystal City III workshop in 2006 also did not list this concern as a topic of discussion. The conference report published 2007 made no mention of it, although it described a wide range of topics discussed at the conference [8].

Over the past 10 years, however, the need for long-term stability testing of co-administered medications or fixed-dose combination drug products has been discussed at several workshops and the controversy has not been completely settled yet. Advantages and disadvantages, practicality, available scientific evidence and other arguments were debated at the annual meetings of Workshop on Recent Issues in Bioanalysis (WRIB) that were held in 2010, 2011, 2012 and 2014 [10-13]. Interest arose mainly on account of some regulators requesting stability data for all co-administered medications in a study. As of now, little evidence has been presented to show drugs in combination have different stability properties that are not identified by short-term stability studies such as freeze- 
thaw and bench-top stability [11-13]. On the other hand, some regulators are of the opinion that since QC and calibrator samples should mimic as closely as possible subject samples, then long-term stability testing of analytes in combination is warranted. US FDA's position on this issue is stated in the 2018 BMV guidance as follows: "For drugs administered as fixed combinations, or part of a specific drug regimen, the stability of the analyte should be assessed in the presence of the other drug. The sponsor should also consider the stability of the analyte in the presence of other co-medications that are known to be regularly administered to patients for the indication of the drug under development" [5]."

\section{Single versus multiple sources for each QC concentration}

In 2015, Health Canada expressed concerns that many drug applicants were conducting matrix-based stability testing using a single tube for each QC concentration which was exposed to storage conditions (stressed) then aliquoted into different tubes prior to processing. This was considered conducting stability testing with $\mathrm{N}=1$, instead of the minimum acceptable approach of $\mathrm{N}=3$, represented by three tubes for each QC concentration stored then processed. The conclusion was that the single tube method for each concentration provides limited information on accuracy, but no information on variability. As such, this method was not considered to be sufficient evidence of stability.

Health Canada issued an announcement ('Clarification of bioanalytical method validation procedures') on 8 October 2015, stating that all method validations conducted with the single tube method were deficient, and validation reports should be amended to include testing utilizing low and high QC aliquoted prior to stability storage [14]. On 9 March 2016, an addendum was issued where the above requirement applied to submissions filed after the October 2015 notice was released [14]. As might be expected, this issue received wide attention among various stakeholders including industry and regulatory agencies. The primary reason: the single tube method was utilized by many firms prior to this issue being raised by Health Canada in 2015. Following discussions with Industry and other regulators, the US FDA initiated a research project in 2016 to collect data and determine possible impact of using a single tube stored then aliquoted and processed, versus three tubes stored then aliquoted and processed. The testing included additional variables as well such as location in the freezer, sample size and different thawing time. This was done for each low and high QC concentration. Model drugs selected included drug products with stability concerns, and one drug that is relatively stable. Preliminary results were presented at the 12th Annual WRIB workshop, April 2018. A manuscript with the final results is currently under preparation.

\section{Conclusion}

The past decade has experienced major advances in science and technology. This is also reflected in the science of bioanalytical methods. Given the much slower pace of regulatory adaptation to the evolving science, conferences such as the Crystal City workshops, WRIB, and others have provided a useful forum for industry, regulators and other scientists to meet and discuss evolving issues in regulated bioanalysis. Although a consensus is not always achieved, the discourse between industry and regulators has contributed much to the understanding of issues and concerns. As regulators gain greater experience and understanding of various scientific issues, they are better positioned to exercise flexibility in the interpretation and application of regulations and guidances. Ultimately, good science should be the goal of all stakeholders, as it provides the foundation for producing accurate and reliable data supporting regulatory decisions for the approval of safe and effective drugs.

\section{Acknowledgements}

The author wishes to thank S Choe and S Kassim for their insightful comments and edits of this commentary.

\section{Disclaimer}

The views expressed in this article do not necessarily represent the official views or policies of the US FDA.

Financial \& competing interests disclosure

The author has no relevant affiliations or financial involvement with any organization or entity with a financial interest in or financial conflict with the subject matter or materials discussed in the manuscript. This includes employment, consultancies, honoraria, stock ownership or options, expert testimony, grants or patents received or pending, or royalties.

No writing assistance was utilized in the production of this manuscript. 


\section{References}

1. US FDA. Guidance for Industry: Bioanalytical Method Validation. US Department of Health and Human Services, US FDA, Center for Drug Evaluation and Research, MD, USA (2001).

2. Shah VP, Midha KK, Dighe SV et al. Analytical methods validation: bioavailability, bioequivalence and pharmacokinetic studies. Pharm. Res. 9(4), 588-592 (1992).

3. Shah VP, Midha KK, Findlay JW et al. Bioanalytical method validation - a revisit with a decade of progress. Pharm. Res. 17(12), 1551-1557 (2000).

4. Shah VP, Bansal S. Historical perspective on the development and evolution of bioanalytical guidance and technology. Bioanalysis 3(8), 823-827 (2011).

5. US FDA. Guidance for Industry: Bioanalytical Method Validation. US Department of Health and Human Services, US FDA, Center for Drug Evaluation and Research, Center for Veterinary Medicine (CVM), MD, USA (2018).

6. European Medicines Agency. Guideline on Bioanalytical Method Validation. EMEA, Committee for Proprietary Medicinal Products (CPMP), London, UK (2011).

7. Jemal M, Ouyang Z, Powell ML. A strategy for a use of incurred biological samples for establishing the acceptability of a liquid chromatography/tandem mass-spectrometric method for quantitation of drugs in biological samples. Rapid Commun. Mass Spectrom 16, 1538-1547 (2002).

8. Viswanathan CT, Bansal S, Booth B et al. Quantitative bioanalytical methods validation and implementation: best practices for chromatographic and ligand binding assays. Pharm. Res. 24(10), 1962-1973 (2007).

9. Fast DM, Kelley M, Viswanathan CT et al. Workshop report and follow-up - AAPS Workshop on Current Topics in GLP Bioanalysis: assay reproducibility for incurred samples - implications of Crystal City recommendations. AAPS J. 11(2), 238-241 (2009).

10. Savoie N, Garofolo F, van Amsterdam P et al. 2010 White Paper on recent issues in regulated bioanalysis and global harmonization of bioanalytical guidance. Bioanalysis 2(12), 1945-1960 (2010).

11. Garofolo F, Rocci M, Dumont I et al. 2011 White Paper on recent issues in bioanalysis and regulatory findings from audits and inspections. Bioanalysis 3(18), 2081-2096 (2011).

12. DeSilva B, Garofolo F, Rocci M et al. 2012 White Paper on recent issues in bioanalysis and alignment of multiple guidelines. Bioanalysis 4(18), 2213-2226 (2012).

13. Fluhler E, Hayes R, Garofolo F et al. 2014 White Paper on recent issues in bioanalysis: a full immersion in bioanalysis (Part 1 - small molecules by LCMS). Bioanalysis 6(2), 3039-3049 (2014).

14. Health Canada. Clarification of bioanalytical method validation procedures. File number: 15-110985-741 (2015). https://www.canada.ca/en/health-canada/services/drugs-health-products/drug-products/announcements/notice -clarification-bioanalytical-method-validation-procedures.html 